\title{
Secondary dysmenorrhea due to a rudimentary, non-communicating functional uterine horn
}

\author{
Ewa Woźniakowska, Anna Stępniak, Piotr Czuczwar, Paweł Milart, Tomasz Paszkowski \\ $3^{\text {rd }}$ Department of Gynecology, Medical University of Lublin, Poland
}

Unicornuate uterus with a rudimentary horn is a rare congenital Müllerian anomaly, which may lead to many obstetrical and gynaecological complications. This pathology occurs in approximately 1/100 000 women. A rudimentary horn forms due to insufficient development of the Müllerian duct. The diagnosis of this anomaly is usually delayed, as it remains asymptomatic until adolescence and its main symptom is dysmenorrhea.

We report a case of a 17-year-old, nulligravida, young woman suffering from progressive, severe dysmenorrhoea for 4 years. Her menstrual cycles were regular with an average flow. The patient had previously been investigated several times in outpatient settings with inconclusive reports and NSAIDs treatment only, without any improvement. The patient was admitted to our department due to deterioration of pelvic pain, during the menstrual period.

The physical examination revealed normal secondary sexual characteristics (Tanner stage M5P5A5) and pain in the right lower abdomen during palpation. The gynaecological examination revealed normal external genitalia and normal vulvar estrogenization. A transvaginal ultrasound and vaginal examination could not be performed because the patient was a virgin.

On transrectal ultrasound examination (TRUS) an unicornuate uterus and normal bilateral ovaries were visualized. Additionally, a right adnexal fluid-filled mass with thick walls isoechoic to the myometrium was seen, separate from the right ovary (Fig. 1). A thin endometrial lining was visualized distinct from the fluid contents and the myometrial wall.

The magnetic resonance imagining (MRI) was also performed. Both TRUS and MRI findings suggested an unicornuate uterus with a hematometra in the right non-communicating rudimentary horn (Fig. 2). No renal abnormalities were observed.

The patient was qualified for a laparotomy. During the procedure an unicornuate uterus with a non-communicating right rudimentary horn dilated to the form of hematometra was seen (Fig. 3), the right fallopian tube was poorly developed and attached distantly to the lateral pelvic wall. The rudimentary horn and residual right fallopian tube were removed (Fig. 4). The post-operative course was uncomplicated. First normal menses occurred on the $28^{\text {th }}$ day after the surgery and was not painful.

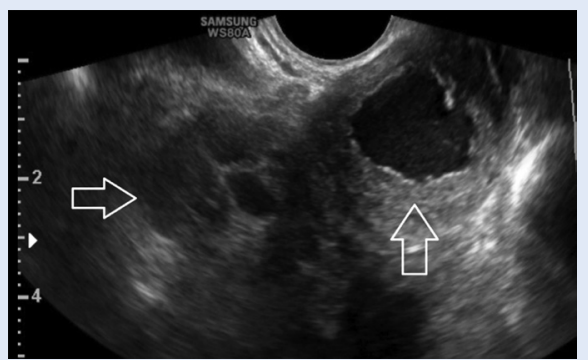

Figure 1. Transvaginal ultrasound image of a rudimentary, non-communicating functional uterine horn (vertical arrow). A normal ovary separate from the mass was also seen (horizontal arrow)

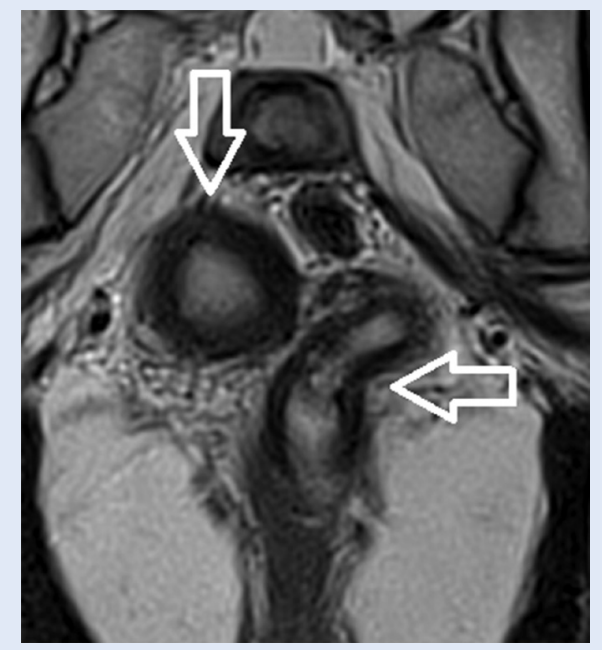

Figure 2. MRI depicted an unicornuate uterus (horizontal arrow) with a rudimentary, noncommunicating uterine horn (vertical arrow). Ovaries were bilaterally unremarkable 


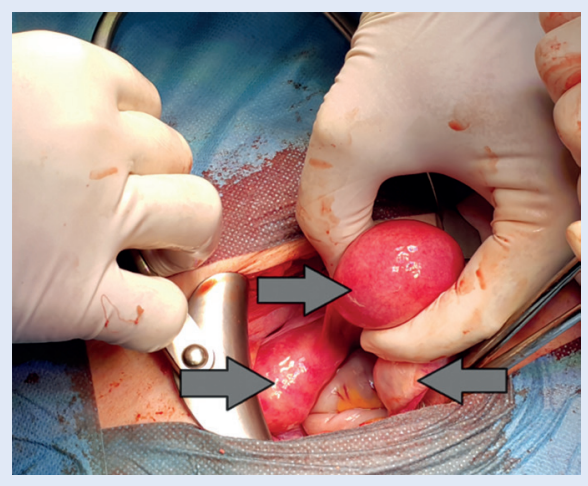

Figure 3. Laparotomy was performed to evacuate the rudimentary, non-communicating uterine horn (top left arrow). A normal right ovary was visualized (right arrow) as well as an unicornuate uterus (bottom left arrow)

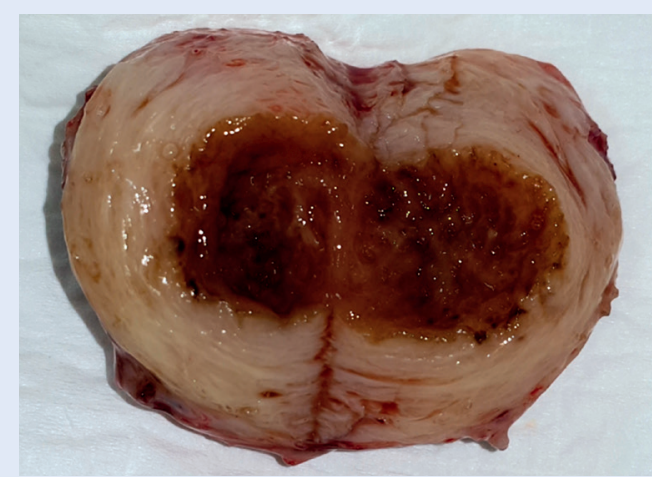

Figure 4. The removed rudimentary, non-communicating uterine horn

The diagnosis of the rudimentary uterine horn is still a challenge. In our case, despite the typical symptoms, the anomaly was not diagnosed for approximately 4 years. It is important to be aware of the possibility of a rudimentary uterine horn in patients with dysmenorrhea. Ultrasound is the most important diagnostic tool in such cases. Surgical removal is the recommended treatment. 